\title{
EL ARTE DE LA DOMINACIÓN: ARTE RUPESTRE Y PAISAJE DURANTE EL PERÍODO INCAICO EN LA CUENCA SUPERIOR DEL RÍO ACONCAGUA
}

\author{
THE ART OF DOMINATION: ROCK ART AND LANDSCAPE IN INKA TIMES IN \\ THE UPPER BASIN OF ACONCAGUA RIVER
}

\author{
Andrés Troncoso M.*
}

\begin{abstract}
Considerando las características formales y espaciales del arte rupestre en la Cuenca Superior del río Aconcagua, Chile central, se aborda la comprensión de la presencia incaica en la zona de estudio y, en especial, el papel jugado por este sistema de representación visual en el proceso de dominio estatal. Para ello, se define la función social del arte rupestre durante el período Intermedio Tardío. Seguidamente, considerando el sentido de esta expresión material y los cambios que lo afectaron durante tiempos incaicos, se analiza el arte rupestre como un agente activo en los procesos sociales de ocupación espacial y dominación implantados por el Tawantinsuyu en la Cuenca Superior del río Aconcagua.
\end{abstract}

Palabras claves: arte rupestre, Cuenca Superior del río Aconcagua, paisaje, semiótica, período Inka.

Considering the formal and spatial characteristics of the rock art in the upper basin of Aconcagua river, central Chile, I attempt to understand the Inka presence in this region and, particularly, the social role played by this system of visual representation. For this purpose, the social function of rock is defined for the Late Intermediate period. Next, considering the meaning of the material statement and the changes that affected it during Inka times, I analize it as an active agent in the social process of spatial occupation and domination imposed by the Tawantinsuyu in the upper basin of the Aconcagua river.

Key words: rock art, upper basin of Aconcagua river, landscape, semiotics, Inka period.

El estudio sobre los procesos de ocupación incaica en sus diferentes provincias ha tendido a concentrarse en tres áreas temáticas distintas que han permitido formular una serie de hipótesis interpretativas para comprender estos procesos: (i) la funcionalidad de la arquitectura incaica y sus características; (ii) la definición del trazado del $q a$ paq ñam y su rol en los procesos sociales prehispánicos, y (iii) las modificaciones en los procesos tecnológicos cerámicos en estos lugares. En este caso, intentaremos ingresar al entendimiento del período incaico en la Cuenca Superior del río Aconcagua (Figura 1) desde lo que podríamos denominar una perspectiva marginal, un enfoque en que la piedra angular para la comprensión del período Tardío en la zona se centre en una materialidad escasamente estudiada en el Tawantinsuyu: el arte rupestre, expresión cultural que adquiere sentido básicamente a partir de su incorporación en un dominio de validez particular y exclusivo (Gallardo 2003; Troncoso 1999), el espacio sociocultural.
Se comprende en esta perspectiva al arte rupestre como una materialidad densa, trabajando desde enfoques interpretativos que centrados en la Arqueología del Paisaje (Criado 1991, 1993) reconocen el rol de la cultura material y el espacio en los procesos sociales y culturales.

\section{Arte Rupestre en el Valle de Aconcagua}

$\mathrm{El}$ arte rupestre, en su técnica de petroglifos, es una de las expresiones materiales más ampliamente conocidas en la zona y de amplia distribución espacial en el área. Asociada generalmente al período Intermedio Tardío (1.000-1.400 d.C.) y agrupada bajo el rótulo de Estilo Aconcagua (Mostny y Niemeyer 1983), las investigaciones efectuadas en el último tiempo han modificado este panorama proponiéndose la presencia de dos estilos de arte rupestre para la Cuenca Superior del Aconcagua (Troncoso 2001a, 2001b, 2002a, 2002b, 2003).

\footnotetext{
* Área de Arqueología, Facultad de Estudios del Patrimonio Cultural, Universidad Internacional SEK. Av. Arrieta 10.000, Peñalolén. atroncos@terra.cl,phandres@usc.es.
} 


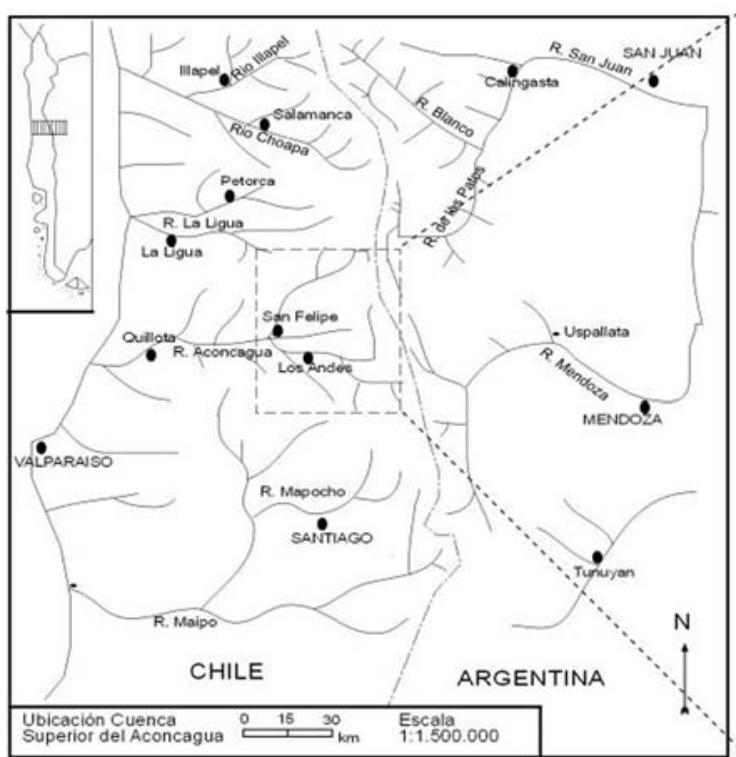

Figura 1. Area de estudio.

The study area.

El primero de ellos, definido como Estilo I de Arte Rupestre para el río Aconcagua, se asociaría al Período Intermedio Tardío y se caracterizaría básicamente por un sistema semiótico que regula toda la producción representacional basado en la aplicación mayoritaria de figuras circulares de tipo compuesta (donde predominan las yuxtaposiciones y apéndices lineales que unen con otras figuras), con una decoración orientada hacia el exterior de la unidad geométrica mayor, un aprovechamiento extensivo del espacio del soporte y una ordenación de las figuras dentro del panel donde predomina una distribución de tipo diagonal dentro del espacio (Figura 2).

En contraposición, el segundo de ellos, o Estilo II de Arte Rupestre del río Aconcagua, se rige por unas pautas diferentes a la anterior y que se resume por un sistema semiótico en el que predomina la presencia de figuras cuadrangulares y ovaladas individuales, con un bajo registro de yuxtaposiciones y una importante frecuencia de superposiciones; la aplicación de decoraciones en el interior de la unidad geométrica mayor, un aprovechamiento intensivo del espacio del soporte y una ordenación de las figuras en el panel de tipo vertical y horizontal (Figura 3). Este sistema semiótico específico del arte rupestre comparte una serie de principios similares con las semióticas específicas de otros sistemas de representación visual de tiempos incaicos, por lo que lo hemos asociado a este momento.

Estas diferencias en el sistema semiótico de cada uno de estos estilos de arte rupestre se reproducen en la distribución espacial diferencial de éstos, siguiendo cada uno pautas particulares de emplazamiento (Troncoso 2002b, 2002c) que le entregan una identidad propia.

Las características del arte rupestre en la Cuenca Superior del Aconcagua posibilitan entonces hablar de dos estilos de arte rupestre basados en códigos semióticos diferentes y particulares a cada uno de ellos. Pero, para entender parte del sentido y el papel jugado por el arte rupestre durante tiempos incaicos, es necesario comprender tanto las características básicas del Intermedio Tardío y Tardío en el área de estudio, así como el rol del arte rupestre y el espacio durante el Intermedio Tardío en la Cuenca Superior del Aconcagua, punto de partida para posteriormente comenzar a aprehender el sentido del arte Inka. 

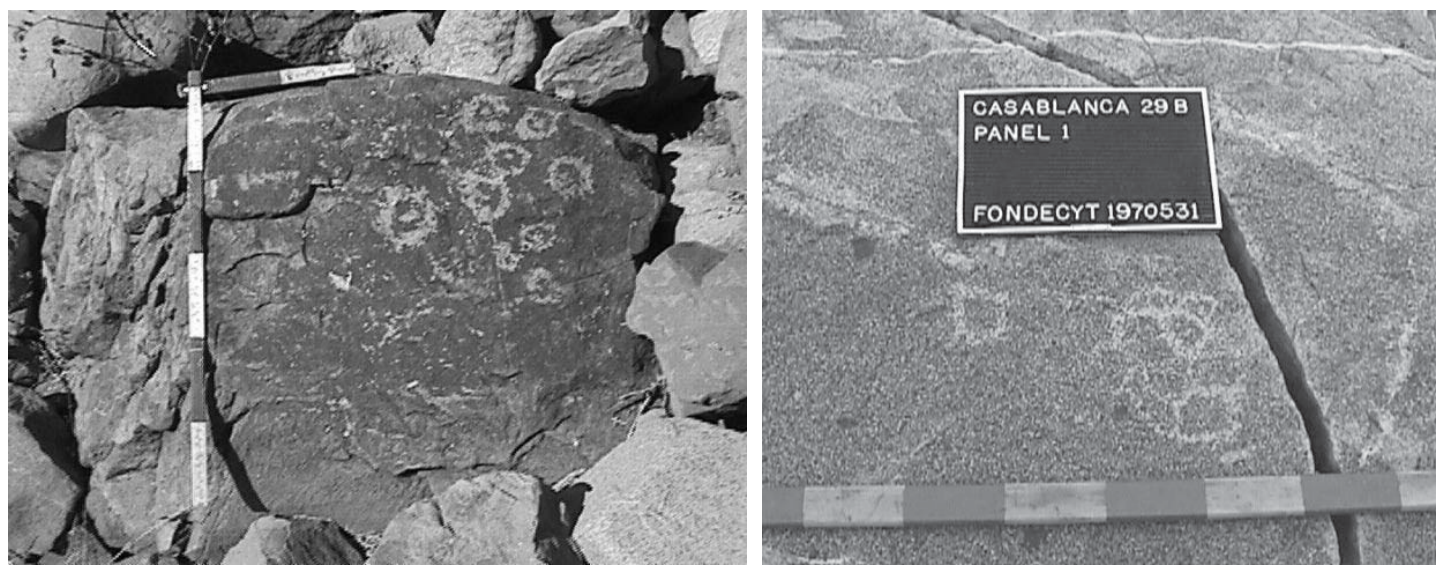

Figura 2. Arte rupestre del período Intermedio Tardío, valle de Putaendo.

Rock art of the Late Intermediate period, Putaendo valley.
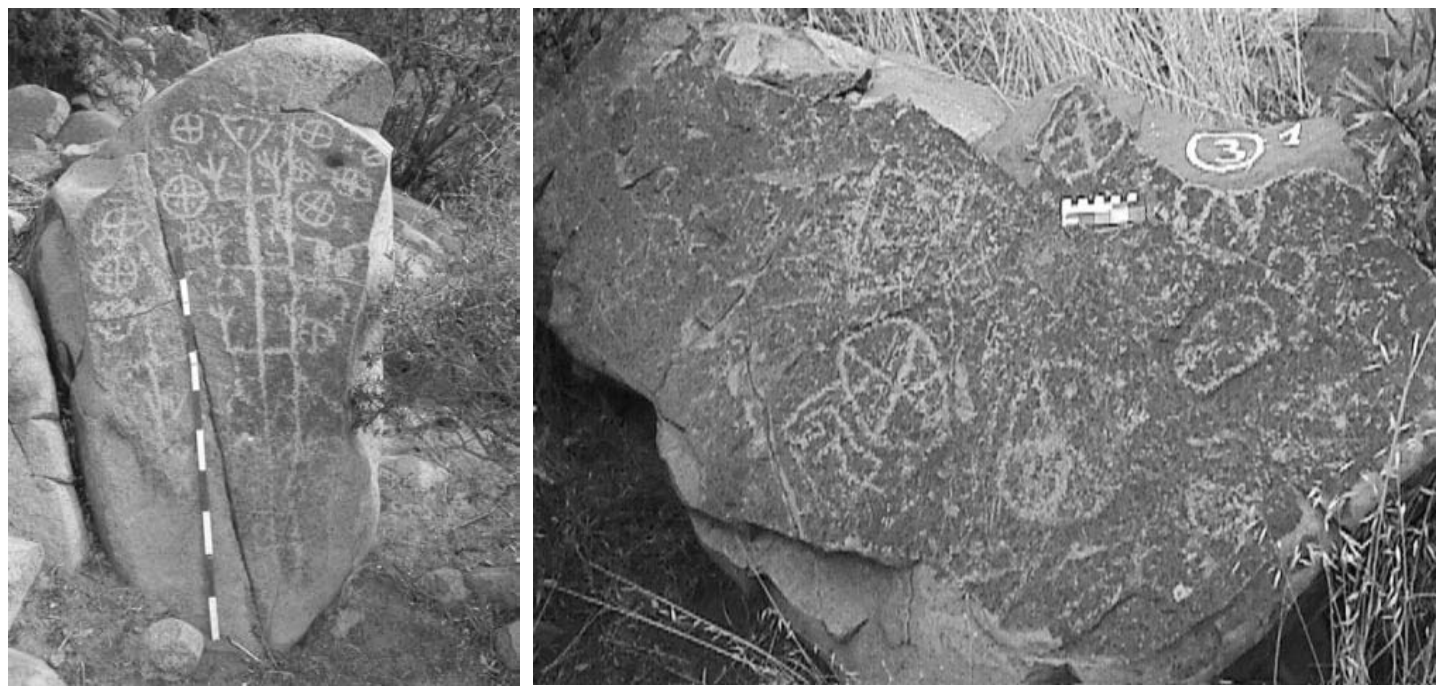

Figura 3. Arte rupestre del período Incaico, valle de Putaendo y cuenca de San Felipe-Los Andes (la numeración de los grabados con pintura no fueron realizados por el autor).

Rock art of the Inka period, valley of Putaendo and San Felipe-Los Andes basin (the numering of the painted images is not by the autor).

\section{Período Intermedio Tardío e Incaico en la Cuenca Superior del Aconcagua}

Dentro de la extensa secuencia de ocupación humana en el área de estudio, que se remonta al menos hasta el período Arcaico (Pavlovic et al. 2002), dos son los momentos que concentran nuestro interés, el Intermedio Tardío e incaico.

El Intermedio Tardío se define por presentar unas particulares características en la Cuenca Superior del Aconcagua, cuales son la ausencia de un único componente cultural en el sector, concibién- dose más bien como un área de interdigitación cultural (Sánchez 2000). En específico, se observa en este espacio la ausencia de los contextos clásicos de la Cultura Aconcagua, siendo reemplazados por contextos locales en los que prima la cerámica Rojo Engobada y se encuentran piezas con decoraciones semejantes a las de la cerámica Diaguita y otras que siguen los lineamientos decorativos de la Cultura Aconcagua, encontrándose una cierta segregación en sus distribuciones, donde las primeras se concentran en el valle de Putaendo y las segundas en la cuenca de San Felipe-Los Andes. 
El período incaico, por su lado, se encuentra ampliamente representado por diferentes instalaciones estatales, entre las que se cuenta el trazado del qapaq ñam, santuario de altura, pucarás, cementerios, wakas y tambos, destacando entre ellos el recientemente descubierto Tambo El Tigre. Todos estos son indicadores de la significativa presencia incaica en este espacio, la cual ha sido entendida dentro de estrategias políticas de anexión y dominación definidas por el Tawantinsuyu en cuanto estado salvaje (Sánchez 2002).

\section{Arte Rupestre, Espacios y Sociedad(es) durante el Período Intermedio Tardío en la Cuenca Superior del Aconcagua}

El Intermedio Tardío marca el comienzo de un importante proceso de monumentalización del espacio en la Cuenca Superior del Aconcagua. Durante este proceso el arte rupestre tuvo, sin lugar a dudas, un rol preponderante y de acción diferencial en las distintas zonas del área de estudio.

Por un lado, durante este tiempo se encuentra una amplia presencia de grabados rupestres en el valle de Putaendo (Tabla 1), los cuales se definen por dos características básicas: encontrarse en los límites de los espacios de ocupación cotidiana de los grupos del período Intermedio tardío y ser ampliamente visibles en el espacio a partir de su emplazamiento en cerros islas, espolones de cerros y laderas, manteniendo entre las distintas estaciones de arte rupestre una visibilidad zonal que permitía a un conocedor del espacio identificar otros puntos con grabados en el entorno.

De esta manera el arte rupestre se orienta a la construcción social del paisaje local por medio de la marcación de los límites de los espacios de la ocupación humana en el valle, definiendo lo que hemos considerado el umbral entre dos tipos de espacio: el espacio doméstico y el espacio salvaje (Troncoso 2002b, 2002c). Pero, por otro lado, el arte rupestre también se constituye en un monumento culturizador del espacio, definiendo una malla de lugares y puntos culturizados en el espacio que posibilitan la construcción de una geografía cultural tanto imaginaria como material en el valle de Putaendo (Troncoso 2002b, 2002c). Y dentro de la construcción de esta geografía cultural, las características particulares de ciertas estaciones de arte rupestre, su asociación con accidentes particulares únicos en Putaendo, cual es una aguada que no se
Tabla 1. Sitios de arte rupestre en el valle de Putaendo. Rock art sites, valley of Putaendo.

\begin{tabular}{lcc}
\hline Sitio & Estilo I & Estilo II \\
\hline Casa Blanca 2 & $\mathrm{P}$ & $\mathrm{A}$ \\
Casa Blanca 3 & $\mathrm{P}$ & $\mathrm{A}$ \\
Casa Blanca 6 & $\mathrm{P}$ & $\mathrm{N} . \mathrm{D}$ \\
Casa Blanca 8 & $\mathrm{P}$ & $\mathrm{A}$ \\
Casa Blanca 13 & $\mathrm{P}(+)$ & $\mathrm{P}$ \\
Casa Blanca 14 & $\mathrm{P}(+)$ & $\mathrm{P}$ \\
Casa Blanca 24 & $\mathrm{P}$ & $\mathrm{A}$ \\
Casa Blanca 26 & $\mathrm{P}$ & $\mathrm{A}$ \\
Casa Blanca 27 & $\mathrm{P}$ & $\mathrm{A}$ \\
Casa Blanca 28 & $\mathrm{P}$ & $\mathrm{A}$ \\
Casa Blanca 29 & $\mathrm{P}$ & $\mathrm{A}$ \\
Casa Blanca 29b & $\mathrm{P}$ & $\mathrm{A}$ \\
Casa Blanca 32 & $\mathrm{P}$ & $\mathrm{A}$ \\
Casa Blanca 33 & $\mathrm{P}$ & $\mathrm{P}(+)$ \\
Casa Blanca 34 & $\mathrm{P}$ & $\mathrm{A}$ \\
Ramadillas 5 & $\mathrm{A}$ & $\mathrm{P}$ \\
Ramadillas 6 & $\mathrm{N} . \mathrm{D}$ & $\mathrm{P}$ \\
Piguchén 2 & $\mathrm{P}$ & $\mathrm{A}$ \\
Piguchén 3 & $\mathrm{P}$ & $\mathrm{A}$ \\
Piguchén 5 & $\mathrm{P}$ & $\mathrm{A}$ \\
Piguchén 6 & $\mathrm{P}$ & $\mathrm{A}$ \\
Tártaro 1 & $\mathrm{P}$ & $\mathrm{P}(+)$ \\
Tártaro 2 & $\mathrm{A}$ & $\mathrm{P}$ \\
Tártaro 3 & $\mathrm{A}$ & $\mathrm{P}$ \\
Tártaro 4 & $\mathrm{A}$ & $\mathrm{P}$ \\
Tártaro 5 & $\mathrm{A}$ & $\mathrm{P}$ \\
Tártaro 7 & $\mathrm{A}$ & \\
\hline & & $\mathrm{P}$ \\
\hline P Presen & &
\end{tabular}

$(\mathrm{P}=$ Presencia, $\mathrm{P}(+)$ = Presencia mayoritaria, $\mathrm{A}=$ Ausencia, N.D. $=$ No determinado).

seca nunca, y su relación con un cementerio tumuliforme del Intermedio Tardío, definen y semantizan espacios únicos y particulares al área, espacios que pueden ser conceptualizados como lugares de amplio capital simbólico y, por tanto, lugares sagrados (Troncoso 2002b, 2002c, 2002d).

En contraposición a lo que ocurre en el valle de Putaendo, la representación del Estilo I de arte rupestre en la cuenca de San Felipe-Los Andes es bastante escasa, reduciéndose a un par de estaciones que se emplazan en las tierras altas del lugar (e.g. Campos de Ahumada) (Troncoso 2002c) o bien en cerros islas como el cerro Paidahuen en la localidad de Los Andes (Coros et al. 2000) (Tabla 2).

Esta mínima representación del arte rupestre en la cuenca de San Felipe-Los Andes y su alta frecuencia en el valle de Putaendo estarían hablando, por tanto, de áreas con prácticas culturales diferentes; un espacio, San Felipe-Los Andes, donde el arte rupestre no es una práctica de amplia frecuencia y, por tanto, no es el medio central de culturización del espacio, y otra zona, el valle de $\mathrm{Pu}$ - 
Tabla 2. Sitios de arte rupestre en la cuenca de San Felipe-Los Andes.

Rock art sites, San Felipe - Los Andes Basin

\begin{tabular}{lcc}
\hline Sitio & Estilo I & Estilo II \\
\hline Campos de Ahumada 1 & N.D. & N.D. \\
Campos de Ahumada 2 & $\mathrm{P}$ & $\mathrm{A}$ \\
Campos de Ahumada 3 & $\mathrm{P}$ & $\mathrm{P}$ \\
Campos de Ahumada 4 & $\mathrm{P}$ & $\mathrm{P}$ \\
Quebrada El Arpa 4 & $\mathrm{P}$ & $\mathrm{P}(+)$ \\
Quebrada El Arpa 5 & $\mathrm{A}$ & $\mathrm{P}$ \\
Quebrada El Arpa 6 & $\mathrm{N} . \mathrm{D}$. & $\mathrm{P}$ \\
Quebrada El Arpa 8 & $\mathrm{A}$ & $\mathrm{P}$ \\
Quebrada El Arpa 9 & $\mathrm{A}$ & $\mathrm{P}$ \\
Quebrada El Arpa 10 & $\mathrm{A}$ & $\mathrm{P}$ \\
Quebrada Honda 1 & $\mathrm{A}$ & $\mathrm{P}$ \\
Quebrada Honda 3 & $\mathrm{A}$ & $\mathrm{P}$ \\
Quebrada Honda 4 & $\mathrm{A}$ & $\mathrm{P}$ \\
Quebrada Honda 6 & $\mathrm{A}$ & $\mathrm{P}$ \\
El Viznagal 1 & $\mathrm{A}$ & $\mathrm{P}$ \\
El Viznagal 2 & $\mathrm{A}$ & $\mathrm{P}$ \\
El Viznagal 3 & $\mathrm{A}$ & $\mathrm{P}$ \\
El Viznagal 4 & $\mathrm{P}$ & $\mathrm{A}$ \\
El Viznagal 6 & $\mathrm{A}$ & $\mathrm{P}$ \\
El Viznagal 7 & $\mathrm{A}$ & $\mathrm{P}$ \\
Cerro Mercachas & $\mathrm{A}$ & $\mathrm{P}$ \\
Cerro Paidahuen & $\mathrm{P}$ & $\mathrm{P}(+)$ \\
El Saino 1 & $\mathrm{N} . \mathrm{D}$. & $\mathrm{P}$ \\
El Saino 2 & $\mathrm{A}$ & $\mathrm{P}$ \\
\hline
\end{tabular}

$(\mathrm{P}=$ Presencia, $\mathrm{P}(+)$ = Presencia mayoritaria, $\mathrm{A}=$ Ausencia, N.D. $=$ No determinado)

taendo, donde el arte rupestre es la principal representación material orientada a la culturización del espacio, redundando en una recurrente presencia. Estas significativas diferencias en la distribución espacial de esta práctica, sumada a variaciones que se dan también en los contextos materiales y tecnológicos entre estas ambas zonas, permiten señalar que durante el Intermedio Tardío nos encontramos ante dos comunidades con identidades diferentes, dos grupos que se definen como entidades diferentes y en el que el arte rupestre sería uno de los principales medio de producción de la identidad social (Troncoso et al. 2003).

Sin embargo, si bien en la cuenca de San Felipe-Los Andes el arte rupestre no sería el principal medio de culturización del espacio, a través de su ínfima presencia, sin duda alguna, estaría marcando y semantizando lugares particulares en la cuenca, espacios posiblemente de gran importancia dentro del imaginario local, tal cual podría haberlo sido el cerro Paidahuén, cerro isla en el que se ha registrado un amplio número de representaciones de arte rupestre de este tiempo.
De esta manera, el arte rupestre jugó un importante papel durante el Intermedio Tardío en los procesos de construcción social de la realidad, definiendo espacios particulares en la geografía de la Cuenca Superior del Aconcagua y (re)produciendo con su presencia-ausencia en el espacio y su producción las identidades particulares de dos comunidades con prácticas culturales e imaginarios propios.

\section{Arte Rupestre, Espacios y Sociedad(es) durante el Período Incaico en la Cuenca Superior del Aconcagua}

La espacialidad del arte rupestre durante el período Incaico sugiere que nos encontramos en este momento ante una nueva realidad en el uso y sentido social de este sistema de representación visual en la Cuenca Superior del Aconcagua (Troncoso 2002a, 2002b, 2002c), uso y sentido que, como en tiempos anteriores, es de acción diferencial en el área de estudio.

Por un lado, encontramos que, a diferencia de lo que ocurría durante el Intermedio Tardío, en el valle de Putaendo el arte rupestre es de muy baja representación espacial, restringiéndose a ciertos espacios del valle. En contraposición, en la cuenca de San Felipe-Los Andes se produce durante este tiempo una explosión en la producción de arte rupestre, realizándose una extensa cantidad de grabados rupestres por los diferentes espacios de este lugar (Tabla 2). De esta forma, durante este momento ocurre una inversión en las prácticas culturales de los grupos locales, inversión por la cual el arte rupestre cambia su orientación espacial dejando de ser una práctica cultural de amplia expresividad en el valle de Putaendo, popularizándose en la cuenca de San Felipe-Los Andes. Así, los espacios de la representación rupestre se modifican substancialmente en la Cuenca Superior del Aconcagua, interactuando el Tawantinsuyu de manera diferencial con este sistema de representación visual en el área, marcando diferencias entre dos espacios de la localidad.

Sin embargo, esta diferenciación que marca el Inka en el espacio de la Cuenca Superior del Aconcagua no es más que el reflejo de una diferenciación proveniente de tiempos anteriores entre dos comunidades distintas. Así, es posible que el Inka haya discriminado la presencia de dos comunidades disímiles en la zona y, por tanto, haya aplicado 
estrategias diferenciales de relación con ellas. En un caso, promoviendo la semantización espacial por medio del arte rupestre y, en el otro, reduciendo la frecuencia de esta práctica cultural.

De hecho, si ampliamos la mirada al resto del contexto de este tiempo, encontramos que estas relaciones diferenciales con estas dos comunidades se refrendan en el uso diferencial de los significantes arquitectónicos, pues mientras en Putaendo la arquitectura se define por la presencia del pucará y su simbolismo relacionado, entre otras cosas, con el uso de la fuerza (Troncoso 2001c, 2002c), siendo una expresión material del poder sobre (Miller y Tilley 1984), en la cuenca de San Felipe-Los Andes, por el contrario, la arquitectura se define más bien por la presencia de instalaciones de tipo religiosos o administrativas burocráticas. Así, se construyen dos espacios marcados por diferencias significativas en su relación con el Inka, un espacio donde la visiblidad de significantes relacionados con el poder físico, y la posibilidad del uso de la violencia, son claramente percibibles (pucará El Tártaro), y otro espacio orientado más hacia la visibilidad de la mitología y el simbolismo del ritual incaico (cerro Mercachas). Y es en este último espacio donde el arte rupestre es ampliamente representado, siendo coherente con la lógica de un espacio plagado de significantes religiosos.

Por otro lado, al observar los espacios micro de representación de los grabados incaicos observamos que ellos adquieren una particular y significativa representatividad espacial en relación a las antiguas estaciones de arte rupestre en la Cuenca Superior del Aconcagua. En el caso del valle de Putaendo, los sitios de arte rupestre de tiempos incaicos se concentran en algunos espacios importantes como son en el Pucará el Tártaro y alrededores, más el espacio definido como área sagrada para el Intermedio Tardío.

En el primer caso, tenemos que los grabados rupestres se disponen en el cordón montañoso del cerro en el que se encuentra el pucará y sus espacios aledaños, sugiriendo posiblemente un proceso de semantización de este particular espacio orientado a la presencia estatal incaica, así como una probable área relacionada con aspectos de ritualidad incaica, como se desprende de las características particulares del sitio Tártaro 4, sitio rupestre consistente en dos grandes rocas contrapuestas que definen un pequeño espacio para la entrada de un individuo y con una distribución diferencial de las imágenes en sus soportes, a un lado predominio de cruces inscritas, al otro predominio de círculos decorados (Troncoso 2002b, 2002c). De hecho, la misma superposición de grabados incaicos por sobre figuras del Intermedio Tardío en una gran roca aledaña al pucará podría sugerir este proceso de resemantización espacial dentro de una nueva lógica representacional.

En el caso de la presencia de grabados rupestres incaicos en el espacio sagrado, se da una distribución bastante particular de estas figuras, las que, si bien son pocas en número, se emplazan en lugares bastante estratégicos de esta área ritual, en su inicio, reocupandando antiguos paneles rupestre (Figura 4); en el centro, en la que sería la principal estación de todo el valle de Putaendo y en superposición a una figura de tiempos previos grabada en el área principal del soporte rocoso y, en el final de este espacio, en el panel que marca el límite de este espacio ritual (Troncoso 2002d). A través de esta particular y específica acción, el arte rupestre incaico se orientaría a la resemantización y reconstrucción de este espacio dentro de la nueva lógica social, posiblemente apropiándose del contenido simbólico de este espacio y dándole una nueva significación.

Esta particular y estratégica distribución espacial del arte rupestre en Putaendo contrasta con la amplia representación del arte rupestre en la cuenca de San Felipe y Los Andes, espacio en el que el arte rupestre se distribuye por las diferentes quebradas y tierras altas de la zona, en especial del sector norte del río Aconcagua.

Se orienta en este espacio el arte rupestre a la semantización de una serie de nuevos espacios, de nuevas áreas que previamente no se encontraban significadas por arte rupestre del Intermedio Tardío. Y es aquí donde se da una de las lógicas que define la distribución espacial de arte rupestre de tiempos incaicos en la Cuenca Superior del Aconcagua: los grabados rupestres incaicos tienden a emplazarse en espacios anteriormente no ocupados por grabados del Intermedio Tardío y, en caso de coexistir grabados de ambos tiempos en un mismo espacio, se da una separación entre paneles con figuras de tiempos incaicos y aquellos con figuras del Intermedio Tardío. En términos generales, las únicas ocasiones en que se produce una coexistencia entre grabados de ambos tiempos es en sitios de características particulares o de importante capital simbólico durante el Intermedio Tardío, tal 


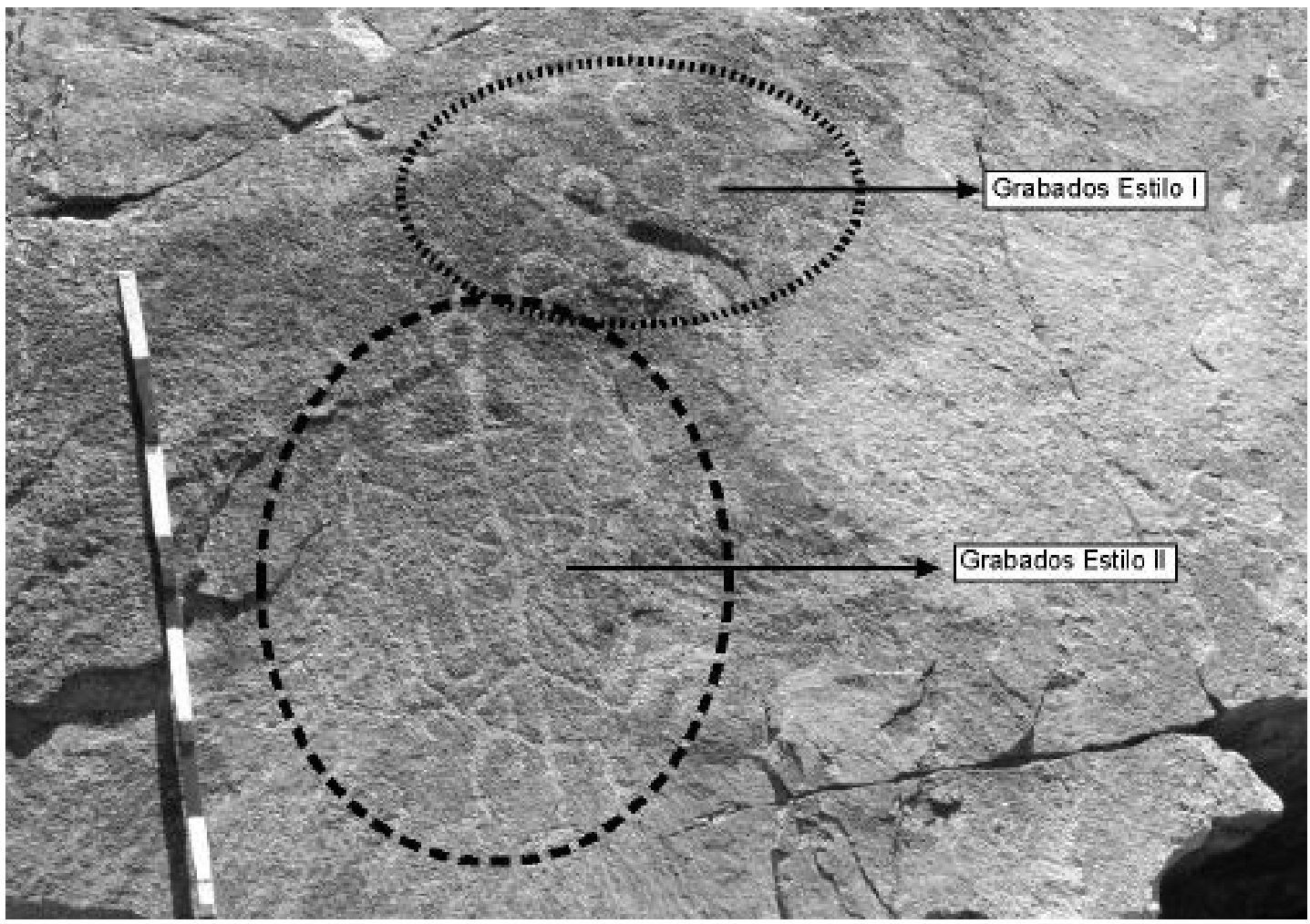

Figura 4. Panel con grabados rupestres de los Estilos I y II, valle de Putaendo.

Rock art Styles I and II, valley of Putaendo.

como lo ejemplifica el caso de Putaendo y que se repite en el caso del cerro Paidahuén, posible espacio de importancia simbólica en el área por ser un cerro isla asociado al río Aconcagua y donde coexisten grabados del período incaico y del Intermedio Tardío.

De esta manera, a partir de su particular distribución espacial y su relación con la espacialidad de otras manifestaciones del Tawantinsuyu, el arte rupestre se orienta a la construcción de un nuevo tipo de paisaje, un espacio que se organiza según criterios diferentes a los de tiempos anteriores y que, por tanto, dan cuenta de una nueva forma de construcción de la realidad en la Cuenca Superior del Aconcagua durante el período Tardío. El arte rupestre define y semantiza nuevos lugares en la geografía local, dándole un nuevo significado a espacios antes construidos por el arte rupestre y construyendo materialmente otras localidades a través de la expresividad representacional del arte rupestre. Así, las tecnologías espaciales incaicas se orientan a lo que Van de Guchte
(1999) considera uno de los principales indicadores de la dominación incaica: la reordenación y resemantización del paisaje local. De hecho, se da una interesante regularidad en esta zona, cual es la asociación entre arte rupestre y asentamientos con arquitectura incaica y amplias condiciones de visibilidad y visibilización en el espacio local (pucará El Tártaro, cerro Mercachas), lo que podría indicar alguna importante relación semántica, social y funcional entre estos dos tipos de sistemas de representación visual, pues de hecho, en sitios con arquitectura sin tales condiciones de dominación visual y espacial, no se han encontrado de momento grabados rupestres, lo que sugiere una exclusividad con los sitios ampliamente visibles en el espacio y que, sin duda alguna, se constituyeron en lugares anclas del paisaje local en tiempos incaicos en la Cuenca Superior del Aconcagua.

Y esta reconstrucción de la geografía local es también un proceso de reorganización de los procesos de construcción social de la realidad, an- 
clando la formulación de un nuevo orden social en una nueva espacialidad, un nuevo orden basado en códigos culturales diferentes a los de tiempos anteriores.

Esta diferencia en los códigos culturales es posible de ser acercada a través del mismo arte rupestre. Si consideramos a esta expresión como una materialidad densa, donde su representatividad no es más que la materialización de una serie de conceptos y principios culturales (Troncoso 2002e), encontramos que las modificaciones que se dan en el sistema semiótico del arte rupestre en tiempos incaicos no son sólo modificaciones de un sistema artístico, sino que son antes que nada, modificaciones de una serie de principios culturales, una alteración tanto del sistema estético local, como de la lógica que guía la producción de este arte y, por tanto, este cambio de estilo da necesaria cuenta de un proceso de modificación cultural, modificación por la cual los criterios estéticos y los conceptos de figuras artísticas, geometría y espacio se han alterado a cabalidad, ingresando la producción de arte rupestre en un nuevo horizonte de inteligibilidad.

$\mathrm{Y}$ este nuevo horizonte de inteligibilidad no es una construcción particular a la Cuenca Superior del Aconcagua, sino que responde a los códigos y principios que regulan el arte Inka a lo largo de todo el Tawantinsuyu (Troncoso 2002c), entrando, por tanto, a funcionar dentro de una lógica acorde a los presupuestos del Tawantinsuyu, una lógica que da cuenta, por un lado, de los principios de ordenamiento de la realidad Incaica y, por otro, de una modificación cultural en la zona que devino en una nueva forma de ordenación social del mundo en la Cuenca Superior del Aconcagua durante el período Tardío, hecho en el que el arte rupestre tuvo un rol preponderante.

\section{Conclusiones}

Comprender los procesos sociales de ocupación y dominación incaica en una región particular es una tarea bastante ardua y extensa, y no pensamos en ningún caso que nuestra perspectiva de cuenta final de este proceso en la Cuenca Superior del Aconcagua. Si creemos que a través de ella es posible comprender cómo la cultura material, y en especial una materialidad tan poco considerada en la arqueología incaica como es el arte rupestre, puede dar cuenta de cambios culturales y sociales durante este tiempo, constituyéndose en un agente activo en los procesos de configuración local de lo social.

En tal sentido, tal vez uno de los puntos importantes a señalar es que la ocupación y dominación del Tawantinsuyu, si bien pudo haber sido ejecutada según diferentes estrategias y reproducida en distintos grados de anexión al Incanato, ella debió orientarse al menos a la construcción de un nuevo orden social, nuevo orden social que requiere, por un lado, el establecimiento de nuevas tecnologías espaciales que permitan construir nuevos paisajes acordes a los criterios semánticos, geopolíticos e institucionales del estado (véase para otros casos Smith 2003), pero requiere también, construir una nueva realidad basada en códigos de comunicación que atraviesen el Tawantinsuyu y permitan mantener un mínimo nivel de comunicación y eficacia a lo largo de su territorio. En tal sentido, el arte rupestre entrega grandes posibilidades para comprender estos procesos, por cuanto esta materialidad requiere la existencia de un código que posibilite la comunicación, pero también un espacio significativo en el cual adquirir sentido, por lo que los cambios producidos en estas dos propiedades de su ser posibilitan acercarse a comprender dinámicas sociales del período Tardío, teniendo siempre como base el conocimiento de la realidad previa a la llegada del Tawantinsuyu, horizonte de comprensión mínima para lograr comprender a cabalidad este proceso.

Así, la creación de un nuevo orden social requiere la acción diferencial y dirigida de la cultura material hacia la producción de ese nuevo orden, pero también la alteración de las relaciones espaciales como forma de lograr construir una nueva realidad cimentada en la bidimensionalidad de la vida social: un discurso material que es referente y se referencia en los discursos inmateriales del Inka.

Finalmente, no podemos dejar de mencionar que si bien el esquema aquí planteado muestra una situación bastante homogénea, creemos que la realidad es bastante más compleja, encontrándose posiblemente una serie de matices en la ocupación Incaica en la Cuenca Superior del Aconcagua, siendo tarea para el futuro intentar avanzar a los aspectos microsociales, y que repercuten en la cotidianeidad, de manera de lograr entender de mejor manera esta compleja realidad sociopolítica en la zona. Asimismo, no podemos dejar de considerar 
que toda esta producción espacial incaica no es más que un discurso político que a expensas de entregar un panorama tan drástico y homogéneo oculte la complejidad de la dinámica social, de las relaciones entre los locales y el Tawantinsuyu, así como la posibilidad del establecimiento de grados diferenciales de anexión y dominio por parte del Inca- nato sobre los grupos locales (Hyslop 1986; Sánchez 2002).

Agradecimientos: A Daniel Pavlovic y Rodrigo Sánchez, compañeros de investigación en la Cuenca Superior del río Aconcagua y al proyecto Fondecyt 1040153.

\section{Referencias Citadas}

Coros, C., C. Coros y A. Garceau

2000 Petroglifos del cerro Paidahuén, Provincia de Los Andes. El Chaski $\mathrm{N}^{\mathrm{o}} 2$.

Criado, F.

1991 Construcción social del espacio y reconstrucción arqueológica del paisaje. Boletín de Antropología Americana 24:5-29.

1993 Visibilidad e interpretación del registro arqueológico. Trabajos de Prehistoria 50:39-56.

Gallardo, F.

2003 Arte rupestre, contenido cultural de la forma e ideología durante el Formativo Temprano en el río Salado (Desierto de Atacama, Norte de Chile). Trabajos en Arqueología del Paisaje (en prensa).

Hyslop. J.

1986 Factors influencing the transmission and distribution of Inka cultural materials throughout Tawantinsuyu. En Latin American Horizons: a Symposium at Dumbarton Oaks, editado por S. Rice, pp: 337-356. Dumbarton Oaks, Washington.

Miller, D. y C. Tilley

1984 Ideology, power and prehistory: an introduction. En Ideology, Power and Prehistory, editado por D. Miller y C. Tilley, pp: 1-15. Cambridge University Press, Cambridge.

Mostny, G. y H. Niemeyer

1983 Arte Rupestre Chileno. Ministerio de Educación, Serie Patrimonio Cultural Chileno, Santiago.

Pavlovic, D., R. Sánchez y A. Troncoso

2002 Patrimonio Cultural del Valle de Aconcagua. Ediciones Centro El Almendral, San Felipe.

Sánchez, R.

2000 Cultura Aconcagua en el valle del río Aconcagua, una discusión sobre su cronología e hipótesis de organización dual. Actas del XIV Congreso Nacional de Arqueología Chilena. Vol 1:147-160. Contribución Arqueológica 5, Museo Regional de Copiapó.

2002 El Tawantinsuyu salvaje en el finis térrea australis. Revista Chilena de Antropología 16:87-107.

Smith, A.

2003 The Political Landscape: Constellations of Authority in Early Complex Polities. University of California Press, California.
Troncoso, A.

1999 De las sociedades en el espacio a los espacios de las sociedades: sobre arqueología y paisaje. Boletín de la Sociedad Chilena de Arqueología 28:37-46.

2001a Rock art in central Chile: forms and styles. International Newsletter on Rock Art 28:6-15.

2001b Sobre el arte rupestre en el curso superior del río Aconcagua y de por qué los signos escudos son Incaicos. Actas del IV Congreso Chileno de Antropología, tomo I: 1392-198. LOM Editores, Santiago.

2001c Espacio y poder. Boletín de la Sociedad Chilena de Arqueología 32:10-23.

2002a Deconstruyendo el signo escudo y el Estilo Aconcagua, reconstruyendo la problemática rupestre en Chile central. Boletín de la Sociedad Chilena de Arqueología 33/34:12-26. 2002b Estilo, arte rupestre y sociedad en la zona central de Chile. Complutum 13:135-153.

2002c Arte rupestre en la Cuenca Superior del Río Aconcagua, zona central de Chile: formas, estilo, espacio y poder. Trabajo de Investigación Tercer Ciclo para optar al Diploma de Estudios Avanzados en Arqueología. Departamento de Historia I, Universidad de Santiago de Compostela, Santiago de Compostela [disponible en www.geocities.com/ arqueo_aconcagua].

2002d Un espacio, tres paisajes, tres sentidos: la configuración de un contexto rupestre en la zona central de Chile. Trabajos en Arqueología del Paisaje (en prensa).

2002e ...A propósito del arte rupestre. Werken 3:67-80.

2003 Proposición de estilos para el arte rupestre del valle de Putaendo, curso superior del río Aconcagua. Chungara Revista de Antropología Chilena 35:209-231.

Troncoso, A.; D. Pavlovic y R. Sánchez

2003 Cultura material, espacialidad, temporalidad y procesos sociales en la Cuenca Superior del río Aconcagua: la configuración de la identidad local durante los períodos Intermedio Tardío, Incaico y el hoy. Ponencia presentada en Identidades en Chile: entre el Pasado y el Presente, entre lo Local y lo Global, Santiago.

van de Guchte, M.

1999 The Inka cognition of landscape: archaeology, etnohistory, and the aesthetics of alterity. En Archaeologies of Landscape: Contemporary Perspectives, editado por W. Ashmore y B. Knapp, pp. 149-168. Blackwell Publishers, Oxford. 